\title{
Von Mäusen und Menschen
}

\author{
Interview: Matthias Scholer
}

Wissenschaftsjournalist

Die Diabetologie erfuhr in den letzten 30 Jahren eine rasante Entwicklung. Wie sich diese stürmische Zeit auf die Forschung und die ärztliche Arbeit auswirkte und welche Lehren daraus gezogen werden sollten, weiss Giatgen Spinas aus eigener Erfahrung. Der Endokrinologe widmete seine ganze berufliche Laufbahn der Erforschung und dem Management der Diabeteserkrankung. Spinas leitete vor seiner Emeritierung 2016 während 20 Jahren die Klinik für Endokrinologie, Diabetologie und Klinische Ernährung des Universitätsspitals Zürich.

Herr Spinas, sie absolvierten kürzlich einen MAS Philosophie und Medizin an der Universität Luzern. Welche Lehren konnten Sie aus diesem Kurs ziehen? Viele. Aber eine der wichtigsten ist sicherlich, dass wir Ärztinnen und Ärzte immer wieder unsere Arbeit und unser Wirken selbstkritisch hinterfragen müssen. Reflexion ermöglicht, den Blickwinkel auf das eigene Handlungsfeld zu erweitern.

$\mathrm{Zu}$ welchen Erkenntnissen in Bezug auf Ihre Tätigkeit als Endokrinologe sind Sie gekommen?

Ich habe erkannt, dass ich die für die akademische Medizin typische Karriere durchlaufen habe, eine Laufbahn im Spannungsfeld zwischen der Notwendigkeit zu publizieren und Forschungsgelder zu akquirieren und einem dem individuellen Wohl der Patienten verpflichteten Berufsethos. Dieses Spannungsfeld bringt es mit sich, dass nicht nur im akademischen Umfeld die Grundanliegen der Medizin, das «cure» und das «care», immer mehr auseinanderdriften.

Sprechen wir zuerst von Ihren Erfahrungen als junger, in der medizinischen Forschung tätiger Mediziner. Sie haben den Publikationsdruck erwähnt. Können sie dies etwas ausführen?

Was für mich in den 1980er Jahren galt, trifft auch heute noch $\mathrm{zu}$. Wenn jemand eine akademische Karriere einschlagen möchte, ist der persönliche Zitations- und h-Index ein ausschlaggebender Faktor bei Bewerbungen. Also stehen die jungen Medizinerinnen und Mediziner unter permanentem Druck, in möglichst hochrangigen Zeitschriften zu publizieren. Darunter leidet schliesslich die Qualität der Forschungsarbeiten. Bis zu 70 Prozent der klinischen Studien ist nicht reproduzierbar und täglich müssen wissenschaft-

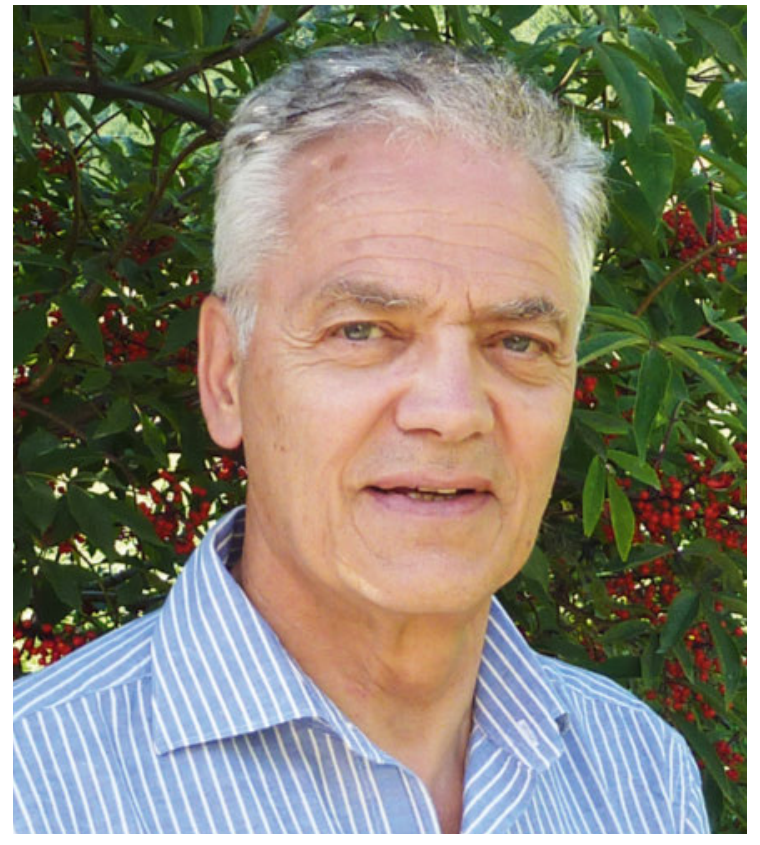

Giatgen Spinas' Abschiedsvorlesung stand im Zeichen einer «diabetosophischen» Rückschau.

liche Arbeiten zurückgezogen werden, weil die Daten manipuliert wurden. Das ist nicht zuletzt das Resultat eines zu stark von persönlichen Zitationsindices und den damit verbundenen «incentives»-getriebenen Wissenschaftsbetriebs. Das eigentliche Ziel der Forschung, epistemischer Erkenntnisgewinn, wird dabei gerne zugunsten einer "guten Story" aus den Augen verloren.

Mit welchem Ziel begannen Sie denn Ihre Forschung? Als ich vor 30 Jahren mit der Diabetologie in Kontakt kam, hatte sich die Erkenntnis durchgesetzt, dass es 
sich bei der heute als «Typ 1» bezeichneten Diabetesform um eine immunvermittelte Krankheit handelt, in deren Verlauf die insulinproduzierenden Betazellen zerstört werden. Ich hatte das Privileg, in einer Gruppe $\mathrm{zu}$ arbeiten, die das sogenannte "Kopenhagener Modell» der Betazellzerstörung entworfen hat. Diese Forschung und die damit verbundenen Publikationen in renommierten Fachzeitschriften gaben meiner Karriere entsprechenden Schub. Viele der damaligen Erkenntnisse beruhten auf dem Modell der «non-obesediabetic mouse», kurz NOD-Maus. Diese galt dazumal im Bereich der Immunologie des Typ-1-Diabetes als «Stein von Rosetta».

\section{Weshalb war diese Inzuchtmaus so bedeutend?}

Die Mäuse passten perfekt zum hypothetischen Modell des Typ-1-Diabetes beim Menschen und erlaubten, die molekularen Abläufe und Mechanismen der Krankheit im Detail zu erforschen. Als man dann noch entdeckte, dass der Diabetes bei den NOD-Mäusen mit einer Blockade des Immunsystems geheilt werden konnte, war die Euphorie schier grenzenlos. Die Heilung einer Typ-1-Diabetes-Erkrankung schien damit auch für Menschen in greifbare Nähe zu rücken. Heute, rund 20 Jahre später, muss man jedoch eine etwas ernüchternde Bilanz ziehen. Sämtliche Versuche, den Diabetes mit einer Immuntherapie zu heilen, verliefen beim Menschen erfolglos. Aufgrund der sehr variablen Pathologie der Insulitis wird es wohl nie eine einfache, für alle Patienten passende Therapie geben. Viel wahrscheinlicher sind personalisierte Ansätze.

\section{Weshalb gelang beim Menschen nicht, was bei Mäusen möglich war?}

Wir müssen feststellen, dass wir uns von der Euphorie über die Züchtung der NOD-Mäuse blenden liessen und nur noch die pathogenetischen Ähnlichkeiten zwischen den Nagern und Menschen sehen wollten. Dabei wurden, zum Teil vielleicht auch bewusst, signifikante Unterschiede im Krankheitsverlauf zwischen den beiden Organismen ignoriert.

\section{Welche Schlussfolgerung ziehen Sie aus dieser Erfahrung?}

Lassen Sie mich mit einem Zitat des Physiologen Claude Bernard antworten: «Si le fait est en opposition avec la théorie, il faut accepter le fait, même si la théorie est soutenue par de grands nom.» Eine kritische Beurteilung der Faktenlage blieb im Fall des NOD-Mausmodells weitgehend aus. Ein berühmter Immunologe bezeichnete die NOD-Maus als «PhDfriendly animal model», dank dem in kurzer Zeit zahlreiche Studien publiziert werden konnten. Wir lernten dabei viel über die molekularen Mechanismen, verloren aber das eigentliche Ziel, nämlich die Zerstörung der Betazellen zu verhindern, aus den Augen.

\section{Dafür wurden im Bereich der Insulinpräparte und der Technologie zur Selbstkontrolle grosse Fortschritte erzielt. Wie stark profitieren die Patienten davon?}

Dank einer Vielzahl beeindruckender Innovationen hat sich nicht nur die Lebensqualität der Patienten und auch die ihrer Angehörigen verbessert. Die Prognose des Krankheitsverlaufs ist nicht mehr mit früheren Jahren vergleichbar. So haben die diabetesbedingte Mortalität und das Risiko, Folgeerkrankungen wie Retinopathie oder Nephropathie zu entwickeln, markant abgenommen. Diese Erfolge wurden zweifelsohne dank modernen Insulinpräparaten möglich, sind aber weitgehend die Folge technologischer Errungenschaften, wie beispielsweise Sensoren für die kontinuierliche Blutzuckermessung, welche die Patienten befähigen, ihre Therapie situationsgerecht und prospektiv zu steuern. Als Schlüsselfaktor für die Verbesserung der Prognose hat sich das ganzheitliche teambasierte interprofessionelle Diabetesmanagement erwiesen - weg vom Paternalismus hin zu selbstbestimmten Patienten.

\section{Interessiert sich die Wissenschaft für den Outcome des Diabetesmanagements?}

Solche Studien fallen unter den Begriff «Versorgungsforschung». Und diese hat es nicht nur in der Diabetologie schwer. Schliesslich geht es dabei oft um schlecht quantifizierbare "soft factors». Entsprechend gering ist das Interesse der hochkarätigen Fachzeitschriften an diesen Themen. Damit fällt automatisch für viele junge Forschende der Anreiz weg, sich in der Versorgungsforschung zu engagieren, da sie, wie erwähnt, ihre Publikationsindizes optimieren müssen. Diese Situation spiegelt sich auch in der Verteilung der Forschungsgelder in der Diabetologie wider. Der weitaus grösste Anteil fliesst in die Grundlagenforschung. Komplexe interprofessionelle Projekte mit unscharfen Variablen hingegen werden nur zögerlich finanziell unterstützt. Aspekte der cure gewinnen so immer mehr an Bedeutung, während solche rund um die care ins Hintertreffen geraten.

Die "conditio humana" ist das Kerngeschäft unserer Profession, auch wenn man medizinische Forschung betreibt.

\section{Was umfasst für Sie der Begriff "care»?}

Im Kern ist care eine deliberative von gegenseitigem Vertrauen getragene Arzt-Patient-Beziehung. Insbesondere bei chronischen lebenslangen Erkrankungen 


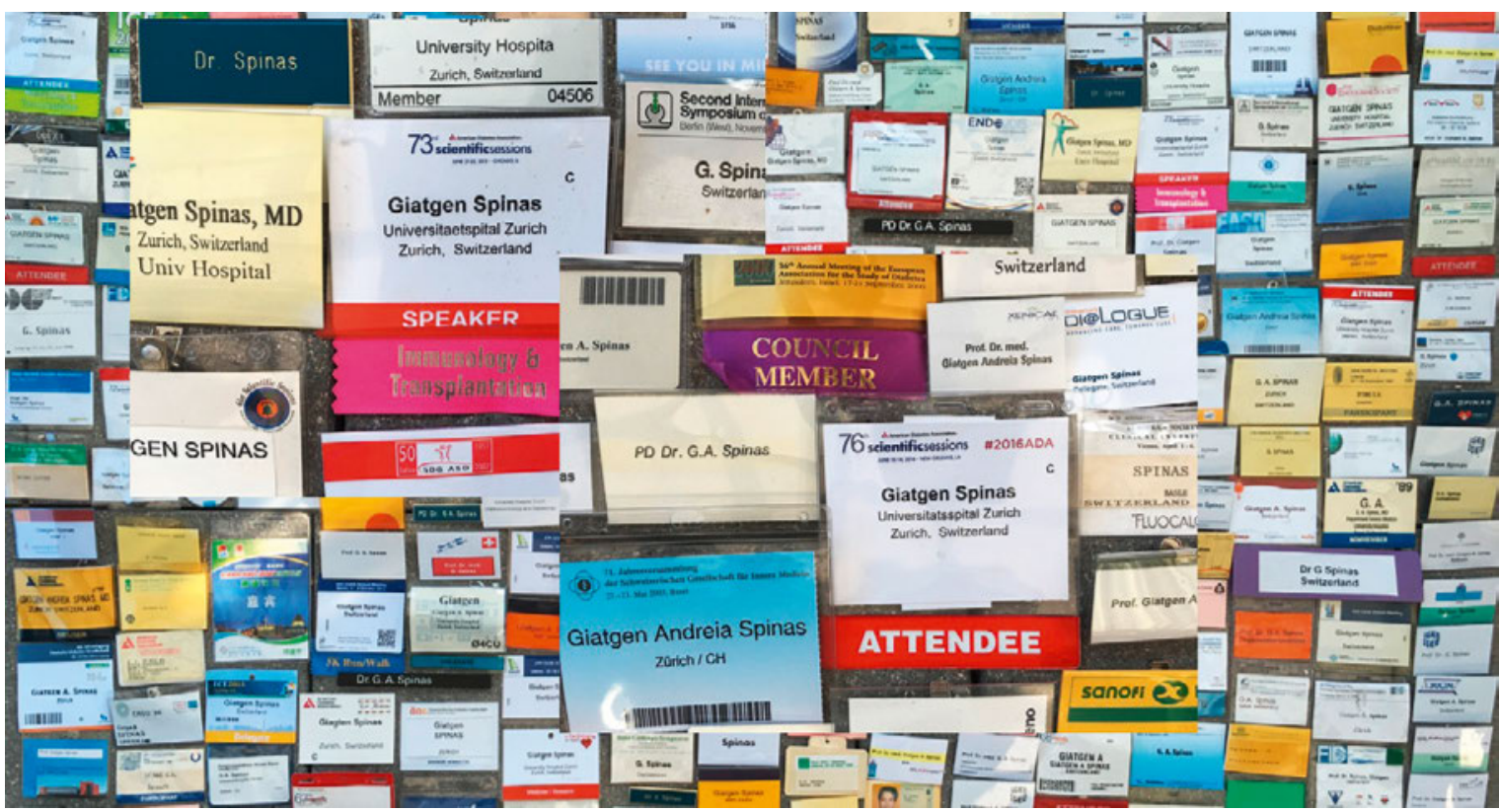

Erinnerungen an eine lange akademische Laufbahn.

wie Diabetes kann sich diese Beziehung phasenweise auch schwierig gestalten. Aber nichtsdestotrotz muss doch das oberste Ziel von uns Ärztinnen und Ärzten das Wohl der uns anvertrauten Patienten sein. Die conditio humana ist das Kerngeschäft unserer Profession, auch wenn man medizinische Forschung betreibt. Denn das Auseinanderklaffen von care und cure wirkt sich bei chronischen Krankheiten wie dem Diabetes katastrophal aus.

\section{Ist dieses Auseinanderdriften eine neue Erscheinung?} Bereits vor zehn Jahren stellte die SAMW in einer Studie zu den Zielen und Aufgaben der Medizin fest, dass Aspekte der cure zu Ungunsten der care überbewertet werden. Damit nicht genug: Eine neuere Erhebung der SAMW an den Hochschulen ergab, dass in der medizinischen Ausbildung der Austausch zwischen cure und care nur in Ansätzen gelebt wird und das ärztliche Ethos nicht im Mittelpunkt der medizinischen Ausund Weiterbildung steht. Diese Erkenntnis führte zur Forderung, medical humanities im Curriculum des Medizinstudiums stärker zu verankern.

\section{Wie kann man den Bruch zwischen care und cure überbrücken?}

Mit Interdisziplinarität und Interprofessionalität. Und das gilt nicht nur für die ärztliche Praxis, sondern auch für die biomedizinische Forschung. Letztere muss weg vom «ich» hin zum «wir». Forschungskonsortien, allenfalls mit Einbezug der Patienten, können ihre Daten früh teilen und gemeinsam definierten Zielen entge-
Giatgen Spinas' Abschiedsvorlesung mit dem Titel «30 Jahre Diabetologie: from cure to care - eine diabetosophische Rückschau" fand im September 2016 statt. Unterlagen zu dieser Ver anstaltung können unter folgender Mailadresse angefordert werden: giatgen.spinas@usz.ch

genarbeiten. So können auch schneller und - wie dies ein think tank der Harvard Medical und der Harvard Business School kürzlich ermittelt hat - auch kostengünstiger Fortschritte im Bereich der cure erreicht werden.

\section{Und im ärztlichen Praxisalltag?}

Der neue Lernzielkatalog für das Medizinstudium, PROFILES, nimmt die gestiegene Bedeutung der Interprofessionalität auf. So soll den künftigen Ärztinnen und Ärzten schon während der Ausbildung eine Haltung disziplinübergreifender Teamarbeit nähergebracht werden. Themen wie Kommunikation und Empowerment der Patienten sollen schon im Bachelorstudium in den Unterricht einfliessen und geübt werden. Alles sinnvolle, zukunftsgerichtete Änderungen. Doch das Wichtigste ist schliesslich, dass man als Ärztin oder Arzt «Menschen mögen muss». Denn, wie es Francis Peabody vor 100 Jahren ausdrückte: «The secret of the care of the patient is in caring for the patient.»

Bildnachweis

(c) Giatgen Spinas 